\title{
The Role of Physical Counselling in Patients with Type 2 Diabetes Mellitus: A Systematic Review
}

\author{
Elisabetta Carraro, Mattia Roppolo, Sara Bonetta, Giorgio Gilli \\ Department of Public Health and Pediatrics, University of Torino, Torino, Italy \\ Email: elisabetta.carraro@unito.it, mattia.roppolo@unito.it, sara.bonetta@unito.it, giorgio.gilli@unito.it
}

Received 24 March 2015; accepted 5 May 2015; published 8 May 2015

Copyright (C) 2015 by authors and Scientific Research Publishing Inc.

This work is licensed under the Creative Commons Attribution International License (CC BY-NC). http://creativecommons.org/licenses/by/4.0/

(c) (1) (9) Open Access

\begin{abstract}
Context: Physical exercise is a key component of treatment and management of people with Type 2 Diabetes, but the role of strategies and interventions to increase and promote physical activity is unclear. Objective: To conduct a systematic review of intervention studies assessing the effects of physical activity counselling on health outcomes in subjects with type 2 diabetes mellitus. Data sources: The Cochrane Library, MEDLINE, EMBASE, CINAHL, LILACS, ClinicalTrials.gov databases were used for the literature search. Data Selection: Studies that assessed the effects of interventions mainly based on physical activity counselling strategies, on physical activity level and glycosylated hemoglobin (HbA1c) were included in the review. Data Extraction: Two independent reviewers extracted the data. Data Synthesis: A total of 23 studies out of 1425 retrieved from databases search, were included in the review. The global number of subjects included in the selected studies was 9913 , and the mean age of participants was $58.8( \pm 8.2)$ years $(\min =46.3 ; \max =73.6)$. The most part of the studies (19) reported values of physical activity level; 13 of them (68.4\%) found a significant effect after the counselling intervention, while $6(31.6 \%)$ did not found significant changes. Among the 17 studies reporting data on HbA1c 9 (52.9\%) described a significant decrease in the counselling intervention group, while $7 \mathbf{( 4 1 . 2 \% )}$ did not find any statistically significant change and $1(5.9 \%)$ reported a significant HbA1c reduction in the comparison group rather than the counselling group. Conclusions: The results presented in this systematic review seem to affirm the usefulness of physical counselling interventions on physical activity and HbA1c, however the lack of homogeneity in the intervention protocols and the contrasting results limit the comprehension of the usefulness of such an approach in patient with type 2 diabetes.
\end{abstract}

\section{Keywords}

Physical Activity Counselling, Interventions, Health Promotion, Lifestyle Change, Type 2 Diabetes Mellitus 


\section{Introduction}

Physical activity is a key component in treatment and management of individuals with type 2 diabetes. The importance and the role of physical activity in type 2 diabetes have been widely reported by previous studies [1]-[3]. Specifically, aerobic and resistance exercises demonstrated their effectiveness in terms of metabolic (glycosylated hemoglobin (HbA1c), fasting blood glucose, blood pressure, glycemic control), physical (muscle strength, endurance, weight, body mass index (BMI), waist circumference, abdominal fat), and psychological (quality of life) indicators [4]-[9].

The current guidelines of the American Diabetes Association and the American College of Sports Medicine suggest 150 minutes per week of moderate-intensity aerobic exercise and three sessions per week of resistance training for patients with type 2 diabetes [10] [11].

Despite the long list of positive effects provided by physical exercises and the current international guidelines, just a minor part of people with type 2 diabetes is physically active. In fact, Thomas and coll. [12] in 2004 reported that only 34\% of people from United Kingdom with diabetes were engaged in physical activity, and 29\% of subjects with type 2 diabetes. Furthermore, only 9\% were involved in activities that may have a positive effect on the health status.

The main barriers to the participation in physical activity can be divided in internal and external [13]. Internal barriers are related to factors, which may depend on personal motivations (i.e., lack of time, lack of motivation), while external barriers are factors independent from the individual's decision-making (i.e., lack of social support, lack of facilities). Both of these barriers categories are to be taken into account in the development and implementation of health-promotion interventions, because they represent the motivation of the scarce engagement in active lifestyle in people with type 2 diabetes.

Data about the engagement in physical exercise and the indications of the barriers that limit the participation to physically demanding activities are the starting points for the development and application of physical activity counselling strategies. Counselling interventions are needed to motivate patients and to involve them in a process of behavioral and lifestyle change, that may have positive effects on their health status and disease management [14].

Counselling practice implies a synergic work from the counselor (nurse, practitioner, kinesiologist, instructor etc.) and the patient. The partnership from the patient and counselor is the first and basic requirement to define difficulties and possibilities to lifestyle change [15]. Then specific counselling strategies can be adopted, but it is necessary that the counselor tries to understand in deep the feelings and difficulties of the patient, in order to suggest and motivate him/her with the most appropriate and tailored indications.

The strategies used to implement physical activity counselling interventions and their effectiveness have not been widely investigated, and for this reason, this study consists in a systematic review of literature of interventional research articles which apply a physical activity counselling intervention on physical activity level and HbA1c in people with Type 2 Diabetes Mellitus. The research question for the article was as follow: is a physical activity counselling intervention effective in terms of physical activity level and HbA1c in people with Type 2 Diabetes?

\section{Methods}

\subsection{Search Strategy and Study Selection}

The following databases were searched for eligible articles without any time restrictions: The Cochrane Library, MEDLINE (by PubMed), EMBASE, CINAHL, LILACS, ClinicalTrials.gov. Moreover, published articles were searched manually. The keywords used in the literature search were: "physical activity counselling", "physical counselling", "counselling", "type 2 diabetes mellitus" and related entry terms. The databases search was not limited for study design or language. The specific research strategy used in PubMed is presented in the Appendix 1.

Only interventional research studies published in English have been considered for this review. All the screening procedure was made and reported in accordance with the Preferred Reporting Items for Systematic Reviews and Meta-Analyses (PRISMA) statement [16].

\subsection{Eligibility Criteria}

In the review were included all the interventional studies that assess the effects of counselling strategies oriented 
to physical activities, in subjects with type 2 diabetes mellitus. The target population was set as adults with type 2 diabetes mellitus. Studies including dietary or exercise co-intervention were also included if the role of physical counselling was highlighted. Studies reporting the effectiveness of counselling strategies in the prevention of type 2 diabetes were not taken into account in the review.

The focus of this review is on physical activity level and HbA1c change, in order to understand the effectiveness of physical counselling on lifestyle modification as well as the primary diabetes-related indicator (HbA1c).

Counselling interventions were defined as specific strategies in which a counselor (practitioner, nurse, peer, kinesiologist etc.) advises, motivates and instructs the patients to regularly participate to exercise, with or without the supplemental use of technological or printed materials specifically designed to support and motivate physical activity in people with type 2 diabetes.

Exclusion criteria were set as following: 1) different population (e.g., patients with type 1 diabetes or gestational diabetes); 2) different research design (e.g., theoretically studies); 3) different or not focused interventions (e.g., lifestyle interventions mainly oriented to pharmacological treatment adherence); 4) duplicate publications.

\subsection{Data Extractions}

The articles resulted from the databases search were independently evaluated by two investigators (E.C. and M.R.). The first step in the revision was the evaluation of title and abstracts of the articles. The two investigators were not blinded to authors, institutions, or manuscript journals.

If the abstract did not provide sufficient information about the eligibility, the inclusion and exclusion criteria, the type and content of the intervention, the studies were included in the full text evaluation.

As a second step of revision, reviewers evaluated independently the full texts of the articles selected in the first round of assessment. Lack of accordance between the reviewers was solved by consensus definition or by a third reviewer (G.G.).

The two reviewers conducted, as a final step in the articles screening, the data extractions. Specifically, the indicators selected were: number of subjects included in the study, mean age of participants, types of interventions proposed (both for intervention and control groups), presence of dietary co-interventions, frequency of the sessions, duration for each session, program duration, dropouts rates, outcomes and results. Disagreements between reviewers were solved by consensus definition or by a third reviewer (G.G.).

\subsection{Quality Assessment}

Quality assessment was made according to the PRISMA recommendation [17] and The Cochrane Collaboration's tool for assessing risk of bias. Study quality assessment included: sequence generation, allocation concealment, blinding of outcomes assessors, incomplete outcome data, selective outcome reporting and other sources of bias.

Quality assessment was independently performed by the two unblinded reviewers. Disagreement between reviewers was resolved through consensus or by a third reviewer (G.G.).

\subsection{Data Analyses}

The current paper is focused on descriptive analysis of the intervention strategies adopted and on qualitative description of the main findings reported in the selected studies.

\section{Results}

\subsection{Description of Studies}

From the 1425 citations retrieved by the electronic databases and manual search, 23 met the inclusion criteria and were included in the review. The search and selection procedure, according to the PRISMA statement [16], is shown in the flow diagram presented in Figure 1.

The total number of subjects included in the selected studies were $9913(\min =8$; $\max =5145)$. The mean age of participants in the 23 selected studies was $58.8( \pm 8.2)$ years $(\min =46.3$; $\max =73.6)$.

The dropouts' rate was reported in 20 studies (87.0\%). The mean dropouts rate was $15.9 \%( \pm 11.9 \%)$, with a 


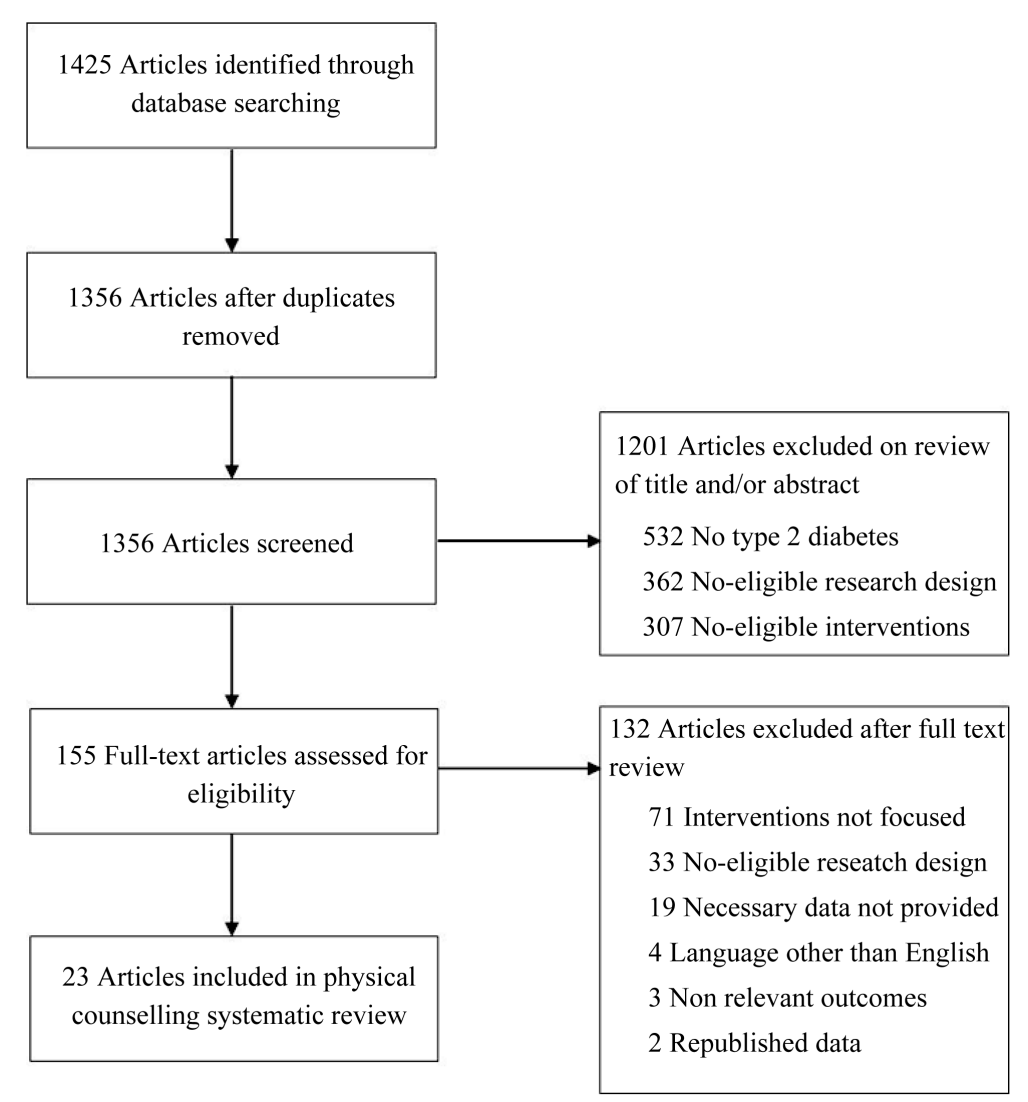

Figure 1. Identification and selection of articles included in the review.

minimum rate of $2.9 \%$ and a maximum of 52\%. The dropouts' rates resulted in line with other similar studies [18]. Characteristics of the studies included in the review are reported in Table 1.

\subsection{Quality Assessment}

The quality assessment among the 23 included studies was as follows: $39.1 \%$ (9 of 23) reported an adequate sequence generation, while $8.7 \%$ (2 of 23) did not report it and 52.2\% (12 of 23) were unclear. $21.7 \%$ of the studies reported allocation concealment ( 5 of 23), 8.7\% did not reported it (2 of 23) and 69.6\% were unclear (16 of 23). Blinding of participants was adequately presented in $4.3 \%$ of the included studies ( 1 of 23$), 17.4 \%$ did not report this information (4 of 23) and 78.3\% were unclear (18 of 23). Incomplete outcome data were adequately addressed in $91.3 \%$ of the studies (21 of 23), while $8.7 \%$ did not addressed this condition (2 of 23). The totality of the studies (100\%) reported an adequate selective outcome reporting. Finally, $17.4 \%$ of the studies (4 of 23) were apparently free from other sources of bias, while 39.1\% did not (9 of 23) and 43.5\% (10 of 23) were unclear about additional sources of bias. Data of quality assessment are reported in Table 2.

\subsection{Description of the Interventions}

The majority of the studies included in the review (87.0\%; 20 of 23) reported an individualized counselling intervention, while $4.3 \%$ (1 of 23) reported group-based counselling activities and $8.7 \%$ (2 of 23) individual and group counselling.

The control group was present in 19 of 23 selected studies (82.6\%). Among these 19 studies $52.6 \%$ (10 of 29) reported usual care for the control group, while 47.4\% (9 of 19) used other types of interventions (e.g. physical activity without counselling; educational sessions; use of printed-based health-related materials).

In 13 of 23 studies (56.5\%) a dietary co-intervention was adopted in association with the physical counselling strategies, while 10 of 23 (43.5\%) did not this co-intervention. Furthermore, 2 of 23 (8.7\%) studies adopted physical activity interventions and 7 of 23 (30.4\%) used printed-based materials, workbook or email messages in 
Table 1. Characteristics of the studies included in the review.

\begin{tabular}{|c|c|c|c|c|c|c|c|c|c|c|c|}
\hline $\begin{array}{l}\text { \& } \\
\vdots \\
0\end{array}$ & $\stackrel{8}{\Xi}$ & $\begin{array}{l}z \\
\text { n } \\
\tilde{E} . \\
\text { के } \\
\text { के }\end{array}$ & 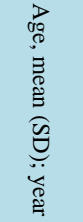 & 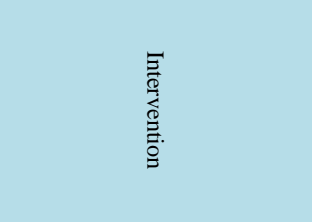 & 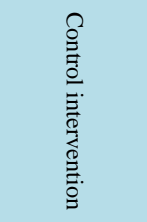 & 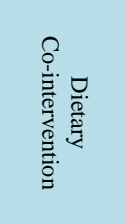 & 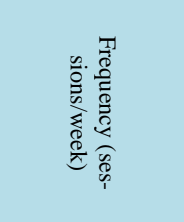 & 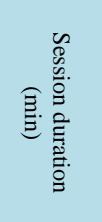 & 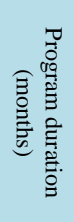 & $\begin{array}{l}Ð \\
0 \\
0 \\
0 \\
心 \\
0 \\
0\end{array}$ & 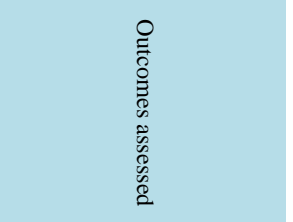 \\
\hline $\begin{array}{c}\text { Batik } \\
\text { et al., } \\
2008[19]\end{array}$ & USA & 14 & $\begin{array}{l}73.6 \\
(7.9)\end{array}$ & $\begin{array}{l}\text { Telephone delivered } \\
\text { motivational program } \\
\text { focused on increasing } \\
\text { physical activity levels }\end{array}$ & Not present & No & $\begin{array}{l}\text { Not } \\
\text { reported }\end{array}$ & $\begin{array}{l}\text { Not } \\
\text { reported }\end{array}$ & 6 & $\begin{array}{l}\text { Not } \\
\text { reported }\end{array}$ & $\begin{array}{c}\text { Physical activity levels; } \\
\text { HbA1c }\end{array}$ \\
\hline $\begin{array}{l}\text { Belalcazar } \\
\text { et al., } \\
2010[20]\end{array}$ & USA & 1759 & $\begin{array}{l}57.5 \\
(5.1)\end{array}$ & $\begin{array}{l}\text { Group and individual } \\
\text { counselling on physical } \\
\text { activity and reductions } \\
\text { of fat intake }\end{array}$ & $\begin{array}{c}\text { Group } \\
\text { sessions } \\
\text { focused on } \\
\text { diet, physical } \\
\text { activity and } \\
\text { social support }\end{array}$ & Yes & $\begin{array}{l}1 \text { session for } \\
\text { week in the first } \\
6 \text { months and } 3 \\
\text { sessions per } \\
\text { month in the } \\
\text { subsequent } 6 \\
\text { months in the } \\
\text { intervention } \\
\text { group } \\
3 \text { sessions per } \\
\text { year in the } \\
\text { control group }\end{array}$ & $\begin{array}{l}\text { Not } \\
\text { reported }\end{array}$ & 12 & 3.2 & $\begin{array}{c}\text { High sensitive C-reactive } \\
\text { protein; weight; BMI; waist } \\
\text { circumference; fasting blood } \\
\text { glucose; HbA1c; LDL } \\
\text { cholesterol; Triglycerides; } \\
\text { HDL cholesterol; physical } \\
\text { fitness }\end{array}$ \\
\hline $\begin{array}{l}\text { Christian } \\
\text { et al., } \\
2008[21]\end{array}$ & USA & 310 & $\begin{array}{c}53.2 \\
(11.0)\end{array}$ & $\begin{array}{l}\text { Individualized tailored report } \\
\text { to improve physical activity } \\
\text { and dietary lifestyle; planning } \\
\text { guide to achieve healthy } \\
\text { lifestyle; a counselling visit } \\
\text { with the physicians about } \\
\text { dietary and physical activity } \\
\text { changes }\end{array}$ & $\begin{array}{l}\text { Health } \\
\text { education } \\
\text { printed } \\
\text { material }\end{array}$ & Yes & $\begin{array}{l}1 \text { session with } \\
\text { the physician } \\
\text { every } 3 \text { months }\end{array}$ & $\begin{array}{l}\text { Not } \\
\text { reported }\end{array}$ & 12 & $\begin{array}{l}9.1 \\
\text { Intervention } \\
\text { group, } \\
14.8 \text { control } \\
\text { group }\end{array}$ & $\begin{array}{l}\text { Physical activity levels; } \\
\text { weight; serum lipids; } \\
\text { HbA1c; caloric intake; } \\
\text { blood pressure; waist } \\
\text { circumference }\end{array}$ \\
\hline $\begin{array}{l}\text { De Greef } \\
\text { et al., } \\
2011[22]\end{array}$ & Belgium & 67 & $\begin{array}{l}67.4 \\
(9.3)\end{array}$ & $\begin{array}{l}\text { (i) Pedometer and diary to } \\
\text { record daily physical activity; } \\
\text { group physical activity coun- } \\
\text { selling delivered by a } \\
\text { behavioral expert (ii) } \\
\text { Pedometer and diary to } \\
\text { record daily physical } \\
\text { activity; individual physical } \\
\text { activity counselling delivered } \\
\text { by th GP }\end{array}$ & $\begin{array}{l}\text { General care } \\
\text { from GP }\end{array}$ & No & $\begin{array}{c}1 \text { session every } 3 \\
\text { weeks }\end{array}$ & $\begin{array}{l}\text { (i) } 90 \\
\text { (ii) } 15\end{array}$ & 3 & $\begin{array}{l}4.7 \text { group } \\
\text { counselling, } \\
8.3 \text { control } \\
\text { group }\end{array}$ & $\begin{array}{l}\text { Pedometer-based physical } \\
\text { activity; self-reported } \\
\text { physical activity; waist } \\
\text { circumference, BMI, } \\
\text { HbA1c; fasting glucose; } \\
\text { total cholesterol }\end{array}$ \\
\hline $\begin{array}{l}\text { Debussche } \\
\text { et al., } \\
2012 \text { [23] }\end{array}$ & $\begin{array}{l}\text { Reunion } \\
\text { Island }\end{array}$ & 398 & $\begin{array}{c}53.8 \\
(11.5)\end{array}$ & $\begin{array}{l}\text { Counselling visits with a } \\
\text { nurse and a dietitian }\end{array}$ & One visit & Yes & $\begin{array}{c}1 \text { session every } 3 \\
\text { months }\end{array}$ & $\begin{array}{l}\text { Not } \\
\text { reported }\end{array}$ & 12 & $\begin{array}{l}26 \\
\text { intervention } \\
\text { group; } 14 \\
\text { control group }\end{array}$ & $\begin{array}{c}\text { HbA1c; BMI; waist } \\
\text { circumference; fat mass; } \\
\text { blood pressure; treatment; } \\
\text { diet; physical activity; } \\
\text { fasting blood glucose; lipid } \\
\text { profile }\end{array}$ \\
\hline $\begin{array}{l}\text { Di Loreto } \\
\text { et al., } \\
2003[14]\end{array}$ & Italy & 340 & $\begin{array}{l}61.8 \\
(0.8)\end{array}$ & $\begin{array}{l}\text { Counseling for diet and } \\
\text { physical activity and } \\
\text { therapeutic prescription; } \\
\text { structured counseling } \\
\text { recommending physical } \\
\text { activity }\end{array}$ & $\begin{array}{l}\text { Counseling } \\
\text { for diet and } \\
\text { physical } \\
\text { activity and } \\
\text { therapeutic } \\
\text { prescription }\end{array}$ & $\begin{array}{l}\text { Yes, for } \\
\text { overweight } \\
\text { and obese } \\
\text { patients }\end{array}$ & $\begin{array}{l}1 \text { session every } 3 \\
\text { t months, and one } \\
\text { phone calls after } \\
\text { the first month }\end{array}$ & 15 & 24 & $\begin{array}{l}1.6 \\
\text { intervention } \\
\text { group }\end{array}$ & $\begin{array}{c}\text { Physical activity level; BMI; } \\
\text { HbA1c }\end{array}$ \\
\hline $\begin{array}{l}\text { Eakin } \\
\text { et al., } \\
2010[24]\end{array}$ & Australia & 434 & $\begin{array}{c}58.2 \\
(11.8)\end{array}$ & $\begin{array}{l}\text { Telephone counselling } \\
\text { focused on physical activity } \\
\text { and healthy diet; workbook of } \\
\text { the activities and pedometer }\end{array}$ & Usual care & Yes & Not reported & 18 & 12 & 21.4 & $\begin{array}{l}\text { Physical activity; \% of } \\
\text { calories from total fat and } \\
\text { saturated fat; grams of fiber } \\
\text { per day; servings of fruit } \\
\text { and vegetables per day }\end{array}$ \\
\hline $\begin{array}{c}\text { Eakin } \\
\text { et al., } \\
2009[25]\end{array}$ & Australia & 434 & $\begin{array}{c}58.2 \\
(11.8)\end{array}$ & $\begin{array}{l}\text { Telephone counselling } \\
\text { focused on physical activity } \\
\text { and healthy diet; workbook of } \\
\text { the activities and pedometer }\end{array}$ & Usual care & Yes & Not reported & $\begin{array}{l}\text { Not } \\
\text { reported }\end{array}$ & 12 & 21.4 & $\begin{array}{l}\text { Physical activity; \% of } \\
\text { calories from total fat and } \\
\text { saturated fat; grams of fiber } \\
\text { per day; servings of fruit } \\
\text { and vegetables per day }\end{array}$ \\
\hline $\begin{array}{c}\text { Eakin } \\
\text { et al., } \\
2014[26]\end{array}$ & Australia & 302 & $\begin{array}{l}58.0 \\
(8.6)\end{array}$ & $\begin{array}{l}\text { Telephone counselling } \\
\text { focused on physical activity } \\
\text { and reducing energy intake; } \\
\text { workbook of the activities }\end{array}$ & Usual care & Yes & $\begin{array}{l}1 \text { session a week } \\
\text { in the first } \\
\text { month; } 1 \text { session } \\
\text { every } 2 \text { weeks } \\
\text { for the } \\
\text { subsequent } 5 \\
\text { months; } 1 \\
\text { session every } \\
\text { month for the } \\
\text { next } 12 \text { months }\end{array}$ & $\begin{array}{l}\text { Not } \\
\text { reported }\end{array}$ & 18 & $\begin{array}{l}26.5 \\
\text { intervention } \\
\text { group; } 20.5 \\
\text { usual care } \\
\quad \text { group }\end{array}$ & $\begin{array}{l}\text { Weight loss; physical } \\
\text { activity; HbA1c; energy } \\
\text { intake; diet quality; waist } \\
\text { circumference; total } \\
\text { cholesterol; HDL } \\
\text { cholesterol; LDL } \\
\text { cholesterol; Total (HDL } \\
\text { cholesterol; triglycerides; } \\
\text { systolic and diastolic blood } \\
\text { pressure }\end{array}$ \\
\hline
\end{tabular}




\section{Continued}

\begin{tabular}{|c|c|c|c|c|c|c|c|c|c|c|c|}
\hline $\begin{array}{c}\text { Eakin } \\
\text { et al., } \\
2013[27]\end{array}$ & Australia & 302 & $\begin{array}{l}58.0 \\
(8.6)\end{array}$ & $\begin{array}{l}\text { Telephone counselling } \\
\text { focused on physical activity } \\
\text { and reducing energy intake; } \\
\text { workbook of the activities }\end{array}$ & Usual care & Yes & $\begin{array}{l}1 \text { session a week } \\
\text { in the first } \\
\text { month; } 1 \text { session } \\
\text { every } 2 \text { weeks } \\
\text { for the } \\
\text { subsequent } 5 \\
\text { months }\end{array}$ & 28.2 & 6 & $\begin{array}{l}12.6 \\
\text { intervention } \\
\text { group; } 7.3 \\
\text { usual care } \\
\text { group }\end{array}$ & $\begin{array}{l}\text { Weight; physical activity; } \\
\text { HbA1c; energy intake; diet } \\
\text { quality }\end{array}$ \\
\hline $\begin{array}{l}\text { Fowles } \\
\text { et al., } \\
2014[28]\end{array}$ & Canada & 180 & $\begin{array}{r}62.4 \\
(10.5) \\
\\
\end{array}$ & $\begin{array}{l}\text { (i) one-to-one physical } \\
\text { activity counselling, } \\
\text { educational workshops and } \\
\text { combined aerobic and } \\
\text { resistance training classes, } \\
\text { provided by a kinesiologist in } \\
\text { a diabetes center; } \\
\text { (ii) exercise counselling and } \\
\text { resistance exercise Instruction } \\
\text { provided by a kinesiologist in } \\
\text { a diabetes center, and exercise } \\
\text { classes organized in a gym; } \\
\text { (iii) exercise counselling and } \\
\text { resistance exercise Instruction } \\
\text { provided by a diabetes } \\
\text { educator and exercise classes } \\
\text { provided by a kinesiologist in } \\
\text { a diabetes center }\end{array}$ & Not present & No & Not reported & $\begin{array}{l}\text { Not } \\
\text { reported }\end{array}$ & 6 & 44.5 & $\begin{array}{l}\text { Physical activity; exercise } \\
\text { participation; perceptions of } \\
\text { efficacy; perceived fitness } \\
\text { and health; weight, fasting } \\
\text { blood glucose; HbA1c }\end{array}$ \\
\hline $\begin{array}{l}\text { Fowles } \\
\text { et al., } \\
2014[29]\end{array}$ & Canada & 198 & $\begin{array}{l}59.0 \\
(10.4)\end{array}$ & $\begin{array}{l}\text { Single counselling } \\
\text { appointment from a } \\
\text { diabetes educator }\end{array}$ & $\begin{array}{l}\text { Single } \\
\text { standard } \\
\text { care } \\
\text { counselling } \\
\text { session }\end{array}$ & No & 1 session & $\begin{array}{l}\text { Not } \\
\text { reported }\end{array}$ & 6 & 52.0 & $\begin{array}{l}\text { Physical activity; exercise } \\
\text { participation; perceptions of } \\
\text { efficacy; perceived fitness } \\
\text { and health; BMI; waist } \\
\text { circumference; systolic and } \\
\text { diastolic blood pressure; } \\
\text { fasting blood glucose; } \\
\text { HbA1c; triglycerides; total } \\
\text { cholesterol; HDL } \\
\text { cholesterol; LDL } \\
\text { cholesterol }\end{array}$ \\
\hline $\begin{array}{c}\text { Gregg } \\
\text { et al., } \\
2012[30]\end{array}$ & USA & 5145 & $\begin{array}{l}58.9 \\
(6.8)\end{array}$ & $\begin{array}{l}\text { Group and individual } \\
\text { counselling on physical } \\
\text { activity and reductions of } \\
\text { fat intake }\end{array}$ & $\begin{array}{l}\text { Group } \\
\text { sessions } \\
\text { focused on } \\
\text { diet, physical } \\
\text { activity and } \\
\text { social support }\end{array}$ & Yes & $\begin{array}{l}1 \text { session for } \\
\text { week in the first } \\
6 \text { months, } 3 \\
\text { sessions per } \\
\text { month in the } \\
\text { subsequent } 6 \\
\text { months and } 2 \\
\text { sessions per } \\
\text { month for the } \\
\text { subsequent } 36 \\
\text { months in the } \\
\text { intervention } \\
\text { group } \\
3 \text { sessions per } \\
\text { year in the } \\
\text { control group }\end{array}$ & $\begin{array}{l}\text { Not } \\
\text { reported }\end{array}$ & 48 & 12.5 & $\begin{array}{l}\text { Fasting blood glucose; } \\
\text { HbA1c }\end{array}$ \\
\hline $\begin{array}{c}\text { Kang } \\
\text { et al., } \\
2010[31]\end{array}$ & $\begin{array}{l}\text { South- } \\
\text { Korea }\end{array}$ & 123 & $\begin{array}{l}46.3 \\
(5.7)\end{array}$ & $\begin{array}{l}\text { Face-to-face counselling } \\
\text { and email lifestyle } \\
\text { program }\end{array}$ & $\begin{array}{l}\text { General } \\
\text { Information } \\
\text { on health }\end{array}$ & Yes & $\begin{array}{l}5 \text { sessions in } 12 \\
\text { weeks and } 1 \\
\text { email every } 3 \\
\text { weeks for } 30 \\
\text { weeks }\end{array}$ & $20-30$ & 24 & $\begin{array}{l}8.7 \text { in the } \\
\text { intervention } \\
\text { group }\end{array}$ & $\begin{array}{l}\text { Weight; BMI; waist } \\
\text { circumference; systolic } \\
\text { and diastolic blood } \\
\text { pressure; fasting blood } \\
\text { glucose; HbA1c; total } \\
\text { cholesterol, LDL choles } \\
\text { terol; HDL cholesterol; } \\
\text { insulin resistance }\end{array}$ \\
\hline $\begin{array}{c}\text { Kirk } \\
\text { et al., } \\
2009[32]\end{array}$ & $\begin{array}{l}\text { United } \\
\text { Kingdom }\end{array}$ & 134 & $\begin{array}{c}61.1 \\
(10.2)\end{array}$ & $\begin{array}{l}\text { (i) Person delivered } \\
\text { physical counselling } \\
\text { (ii) Written delivered } \\
\text { physical counselling }\end{array}$ & Usual care & No & $\begin{array}{l}6 \text { sessions in } 12 \\
\text { months }\end{array}$ & (i) 30 & 12 & $\begin{array}{l}\text { (i) } 10.6 \\
\text { intervention } \\
\text { group; } \\
\text { (ii) } 17.3 \\
\text { intervention } \\
\text { group; } \\
\text { 11.4 control } \\
\text { group }\end{array}$ & $\begin{array}{l}\text { Physical activity level; } \\
\text { HbA1c; total cholesterol; } \\
\text { HDL cholesterol; } \\
\text { medication; waist } \\
\text { circumference; BMI; } \\
\text { systolic and diastolic } \\
\text { blood pressure }\end{array}$ \\
\hline $\begin{array}{c}\text { Kirk } \\
\text { et al., } \\
2004[33]\end{array}$ & $\begin{array}{l}\text { United } \\
\text { Kingdom }\end{array}$ & 70 & $\begin{array}{l}57.6 \\
(7.9)\end{array}$ & $\begin{array}{l}\text { One-to-one physical } \\
\text { activity counselling }\end{array}$ & Usual care & No & $\begin{array}{l}2 \text { sessions of } \\
\text { counselling and } \\
4 \text { follow-up calls } \\
\text { in } 12 \text { months }\end{array}$ & 30 & 12 & $\begin{array}{l}14.3 \\
\text { intervention } \\
\text { group; } \\
\text { 17.1 control } \\
\text { group }\end{array}$ & $\begin{array}{l}\text { Physical activity; BMI, } \\
\text { blood pressure; HbA1c; } \\
\text { total cholesterol; HDL } \\
\text { cholesterol; LDL } \\
\text { cholesterol; triglycerides; } \\
\text { fibrinogenon; t-PA }\end{array}$ \\
\hline
\end{tabular}




\section{Continued}

\begin{tabular}{|c|c|c|c|c|c|c|c|c|c|c|c|}
\hline $\begin{array}{c}\text { Kirk } \\
\text { et al., } \\
2004[34]\end{array}$ & $\begin{array}{l}\text { United } \\
\text { Kingdom }\end{array}$ & 70 & $\begin{array}{l}57.6 \\
(7.9)\end{array}$ & $\begin{array}{l}\text { One-to-one physical } \\
\text { activity counselling }\end{array}$ & Usual care & No & $\begin{array}{l}2 \text { sessions of } \\
\text { counselling and } \\
4 \text { follow-up calls } \\
\text { in } 12 \text { months }\end{array}$ & 30 & 12 & $\begin{array}{c}14.3 \\
\text { intervention } \\
\text { group; } \\
\text { 17.1 control } \\
\text { group }\end{array}$ & $\begin{array}{l}\text { Physical activity; stage of } \\
\text { exercise behavior change; } \\
\text { processes of exercise } \\
\text { behavior change }\end{array}$ \\
\hline $\begin{array}{c}\text { Look } \\
\text { ahead } \\
\text { group, } \\
2014 \\
{[35]}\end{array}$ & USA & 5145 & $\begin{array}{l}58.6 \\
(6.8)\end{array}$ & $\begin{array}{l}\text { Group and individual } \\
\text { counselling on physical } \\
\text { activity and reductions of fat } \\
\text { intake }\end{array}$ & $\begin{array}{c}\text { Group } \\
\text { sessions } \\
\text { focused on } \\
\text { diet, physical } \\
\text { activity and } \\
\text { social support }\end{array}$ & Yes & $\begin{array}{l}1 \text { session for } \\
\text { week in the first } \\
6 \text { months, } 3 \\
\text { sessions per } \\
\text { month in the } \\
\text { subsequent } 6 \\
\text { months and } 2 \\
\text { sessions per } \\
\text { month for the } \\
\text { subsequent } 84 \\
\text { months in the } \\
\text { intervention } \\
\text { group } \\
3 \text { sessions per } \\
\text { year in the first } \\
48 \text { months and } \\
\text { one session per } \\
\text { year in the } \\
\text { subsequent } 48 \\
\text { months in the } \\
\text { control group }\end{array}$ & $\begin{array}{l}60-75 \\
\text { group } \\
\text { sessions; } \\
20 \text { - 30 } \\
\text { individual } \\
\text { sessions }\end{array}$ & 96 & $\begin{array}{c}10.1 \\
\text { intervention } \\
\text { group; } \\
11.7 \text { control } \\
\text { group }\end{array}$ & $\begin{array}{l}\text { Weight; physical activity } \\
\text { level; calories and fat } \\
\text { intake; meal replacement; } \\
\text { monitored weight }\end{array}$ \\
\hline $\begin{array}{l}\text { Plotnikoff } \\
\text { et al., } \\
2013 \\
{[36]}\end{array}$ & Canada & 287 & $\begin{array}{c}61.6 \\
(11.8)\end{array}$ & $\begin{array}{l}\text { (i) standard physical activity } \\
\text { educational material and } \\
\text { stage-based, print materials } \\
\text { developed to address issues } \\
\text { specific to the physical } \\
\text { activity stage in which they } \\
\text { were currently assessed } \\
\text { (ii) standard physical activity } \\
\text { educational material and } \\
\text { stage-based, print materials } \\
\text { developed to address issues } \\
\text { specific to the physical } \\
\text { activity stage in which they } \\
\text { were currently assessed plus } \\
\text { telephone counselling }\end{array}$ & $\begin{array}{l}\text { Standard } \\
\text { physical } \\
\text { activity } \\
\text { educational } \\
\text { material }\end{array}$ & No & $\begin{array}{l}\text { (ii) } 1 \text { call per } \\
\text { week in the first } \\
\text { month; } 2 \text { call per } \\
\text { month in the } \\
\text { subsequent } \\
\text { month and } 1 \text { call } \\
\text { per month for } \\
\text { the next } 10 \\
\text { months }\end{array}$ & (ii) 15 & 12 & $\begin{array}{l}\text { (i) } 33.0 \\
\text { intervention } \\
\text { group; } \\
\text { (ii) } 29.2 \\
\text { intervention } \\
\text { group } \\
16.0 \text { control } \\
\text { group }\end{array}$ & $\begin{array}{c}\text { Physical activity level; } \\
\text { HbA1c; BMI; c-reactive } \\
\text { protein; blood insulin; } \\
\text { fasting blood glucose; HDL } \\
\text { cholesterol; LDL } \\
\text { cholesterol; triglycerides; } \\
\text { HRQOL }\end{array}$ \\
\hline $\begin{array}{l}\text { Plotnikoff } \\
\text { et al., } \\
2011 \text { [37] }\end{array}$ & Canada & 96 & 60 & $\begin{array}{l}\text { Diabetes education program } \\
\text { and individualized physical } \\
\text { activity counselling and } \\
\text { prescription }\end{array}$ & $\begin{array}{l}\text { Diabetes } \\
\text { education } \\
\text { program }\end{array}$ & No & $\begin{array}{l}2 \text { sessions per } \\
\text { week, control } \\
\text { group; } \\
2 \text { sessions per } \\
\text { week in the first } \\
4 \text { weeks, two } \\
\text { sessions per } \\
\text { month in the } \\
\text { subsequent } 10 \\
\text { week and one } \\
\text { session per } \\
\text { month in the } \\
\text { next } 10 \text { weeks, } \\
\text { intervention } \\
\text { group }\end{array}$ & $\begin{array}{l}\text { Not } \\
\text { reported }\end{array}$ & 6 & $\begin{array}{c}12.2 \text { control } \\
\text { group; } \\
4.3 \\
\text { intervention } \\
\text { group }\end{array}$ & $\begin{array}{l}\text { Physical activity level; } \\
\text { HbA1c; aerobic fitness; } \\
\text { fasting blood glucose; } \\
\text { c-reactive protein; } \\
\text { microalbumin; albumin to } \\
\text { creatine ratio; CV risk } \\
\text { factors; insulin; oral } \\
\text { antidiabetic agents }\end{array}$ \\
\hline $\begin{array}{l}\text { Plotnikoff } \\
\text { et al., } \\
2010[38]\end{array}$ & Canada & 8 & $\begin{array}{l}59.5 \\
(6.5)\end{array}$ & $\begin{array}{c}\text { Peer physical activity } \\
\text { counselling delivered by } \\
\text { phone }\end{array}$ & Not present & No & 1 per week & $10-15$ & 3 & Not reported & $\begin{array}{l}\text { Qualitative data on physical } \\
\text { activity; physical activity } \\
\text { related social, cognitive and } \\
\text { behavioral measures; }\end{array}$ \\
\hline $\begin{array}{l}\text { Wadden } \\
\text { et al., } \\
2009 \text { [39] }\end{array}$ & USA & 5145 & $\begin{array}{l}58.6 \\
(6.8)\end{array}$ & $\begin{array}{l}\text { Group and individual } \\
\text { counselling on physical } \\
\text { activity and reductions of fat } \\
\text { intake }\end{array}$ & $\begin{array}{c}\text { Group } \\
\text { sessions } \\
\text { focused on } \\
\text { diet, physical } \\
\text { activity and } \\
\text { social support }\end{array}$ & Yes & $\begin{array}{l}1 \text { session for } \\
\text { week in the first } \\
6 \text { months, } 3 \\
\text { sessions per } \\
\text { month in the } \\
\text { subsequent } 6 \\
\text { months in the } \\
\text { intervention } \\
\text { group } \\
3 \text { sessions per } \\
\text { year in the } \\
\text { control group }\end{array}$ & $\begin{array}{l}60 \text { - } 75 \\
\text { group } \\
\text { sessions; } \\
20 \text { - 30 } \\
\text { individual } \\
\text { sessions }\end{array}$ & 12 & $\begin{array}{l}2.9 \\
\text { intervention } \\
\text { group; } 4.3 \\
\text { control group }\end{array}$ & $\begin{array}{l}\text { Weight; behavioral } \\
\text { adherence }\end{array}$ \\
\hline $\begin{array}{l}\text { Yoo et al., } \\
2007 \text { [40] }\end{array}$ & $\begin{array}{l}\text { South- } \\
\text { Korea }\end{array}$ & 48 & $\begin{array}{l}55.2 \\
(7.3)\end{array}$ & $\begin{array}{c}\text { Education and counselling on } \\
\text { diet and exercise }\end{array}$ & $\begin{array}{l}\text { Educational } \\
\text { group session }\end{array}$ & Yes & $\begin{array}{l}1 \text { session per } \\
\text { week }\end{array}$ & 60 & 9 & $\begin{array}{l}\text { Not } \\
\text { reported }\end{array}$ & $\begin{array}{l}\text { Fasting blood glucose; } \\
\text { HbA1c; weight; BMI; } \\
\text { visceral fat }\end{array}$ \\
\hline
\end{tabular}


Table 2. Quality assessment of the studies included.

\begin{tabular}{|c|c|c|c|c|c|c|}
\hline Source & $\begin{array}{l}\text { Sequence } \\
\text { generation }\end{array}$ & $\begin{array}{l}\text { Allocation } \\
\text { concealment }\end{array}$ & $\begin{array}{l}\text { Blinding of } \\
\text { participants }\end{array}$ & $\begin{array}{l}\text { Incomplete } \\
\text { outcome data }\end{array}$ & $\begin{array}{l}\text { Selective } \\
\text { outcome } \\
\text { reporting }\end{array}$ & $\begin{array}{l}\text { Other sources } \\
\text { of bias }\end{array}$ \\
\hline Batik et al., 2008 [19] & Unclear & Unclear & Unclear & No & Yes & No \\
\hline Belalcazar et al., 2010 [20] & Unclear & Unclear & Unclear & Yes & Yes & Unclear \\
\hline Christian et al., 2008 [21] & Unclear & Unclear & No & Yes & Yes & Unclear \\
\hline De Greef et al., 2011 [22] & Yes & Yes & No & No & Yes & No \\
\hline Debussche et al., 2012 [23] & Yes & Unclear & Unclear & Yes & Yes & Unclear \\
\hline Di Loreto et al., 2003 [14] & Unclear & Unclear & Unclear & Yes & Yes & Unclear \\
\hline Eakin et al., 2010 [24] & Unclear & Unclear & Unclear & Yes & Yes & Unclear \\
\hline Eakin et al., 2009 [25] & Unclear & Unclear & Unclear & Yes & Yes & Unclear \\
\hline Eakin et al., 2014 [26] & Yes & Unclear & Unclear & Yes & Yes & No \\
\hline Eakin et al., 2013 [27] & Yes & Unclear & Unclear & Yes & Yes & No \\
\hline Fowles et al., 2014 [28] & No & No & No & Yes & Yes & No \\
\hline Fowles et al., 2014 [29] & No & No & No & Yes & Yes & No \\
\hline Gregg et al., 2012 [30] & Unclear & Unclear & Unclear & Yes & Yes & Unclear \\
\hline Kang et al., 2010 [31] & Unclear & Unclear & Unclear & Yes & Yes & No \\
\hline Kirk et al., 2009 [32] & Yes & Yes & Yes & Yes & Yes & Unclear \\
\hline Kirk et al., 2004 [33] & Yes & Yes & Unclear & Yes & Yes & Yes \\
\hline Kirk et al., 2004 [34] & Yes & Yes & Unclear & Yes & Yes & Yes \\
\hline Look ahead group, 2014 [35] & Unclear & Unclear & Unclear & Yes & Yes & Yes \\
\hline Plotnikoff et al., 2013 [36] & Yes & Yes & Unclear & Yes & Yes & Unclear \\
\hline Plotnikoff et al., 2011 [37] & Yes & Unclear & Unclear & Yes & Yes & Unclear \\
\hline Plotnikoff et al., 2010 [38] & Unclear & Unclear & Unclear & Yes & Yes & No \\
\hline Wadden et al., 2009 [39] & Unclear & Unclear & Unclear & Yes & Yes & Yes \\
\hline Yoo et al., 2007 [40] & Unclear & Unclear & Unclear & Yes & Yes & No \\
\hline$\%$ Yes & 39.1 & 21.7 & 4.3 & 91.3 & 100 & 17.4 \\
\hline$\%$ No & 8.7 & 8.7 & 17.4 & 8.7 & - & 39.1 \\
\hline \% Unclear & 52.2 & 69.6 & 78.3 & - & - & 43.5 \\
\hline
\end{tabular}

association with counselling strategies.

The frequency of the counselling sessions was very heterogeneous and variable among the studies. Specifically, as extreme values, it was found that Fowles and colleagues [29] analyzed the effects of a single session of counselling, while other authors [38]-[40] adopted a one session per week strategy. However, no consensus or a single approach is identifiable in the frequency of treatments. The session’s duration had a mean value of 33.6 $( \pm 23.1)$ minutes with a minimum of 10 minutes [38] and a maximum of 90 minutes [22]. The counselling session duration was not reported by $39.1 \%$ (9 of 23) of the studies.

Finally, large variability was found also in the program duration. The mean duration was $16.3( \pm 19.8)$ months, with a minimum value of 3 months [22] and a maximum of 96 months [35].

\subsection{Description of the Outcomes}

Several outcomes were analyzed in the selected studies. Among them, the most commonly treated were: physical activity level (82.6\%, 19 of 23); HbA1c (73.4\%, 17 of 23); BMI (43.5\%, 10 of 23); weight (39.1\%, 9 of 23); 
fasting blood glucose (39.1\%, 9 of 23); cholesterol (34.8\%, 8 of 23); waist circumference (34.8\%, 8 of 23) and blood pressure (30.4\%, 7 of 23).

Also in the case of outcomes, the selected studies, reported a lack of homogeneity and none of the principal health-outcomes connected to type 2 diabetes mellitus were reported in all of the articles.

\subsection{Associations of Physical Counselling with Primary Outcomes}

This analysis is focused on the qualitative descriptions of the selected articles in relation to the primary outcomes of this review (physical activity level and HbA1c).

Physical Activity Level. From the 19 included studies reporting values of physical activity level, 13 of them (68.4\%) described a significant effect after the implementation of the counselling intervention, while 6 (31.6\%) did not found significant changes.

More specifically, Christian and colleagues [21] found that, after their 12 months program on dietary and physical activity changes counselling, participants of the intervention group increased the recommended level of physical activity from $26 \%$ to 53\%, while the control group remained stable. DeGreef and colleagues [22] demonstrated an increase of both objective-measured (pedometer) and self-reported physical activity level, after the application of 3 months physical counselling delivered by a behavioral expert or general practitioner. Di Loreto and coll. [14] reported that, after 24 months structured counseling recommending physical activity, the $69 \%$ of participants allocated in the intervention group achieved the objective of $10 \mathrm{MET} /$ day based on physical activity, while just the $18 \%$ of the control group reach a similar result. Eakin and collegues [24] [25] demonstrated the effectiveness of a 12 months telephone delivered counselling intervention with the use, also, of a workbook of the activities, in terms of physical activity level (time and sessions of physical activity) [24] and moderate-tovigorous physical activity level [25]. In two further studies, Eakin and coll. [27] found an increasing in physical activity level in the intervention group, in which a 6 months telephone delivered counselling intervention was implemented, in comparison with a control group, which undertake usual care. The continuation of this study [26] reported the effects of the intervention delivered for 18 months, describing the effectiveness in terms of moderate-to-vigorous physical activity on the intervention group, which was $42 \%$ higher than the control group. Fowles and collegues [28] in their 6 months intervention study described an increase of the counselling group in terms of exercise participation, but a non-significant change in terms of moderate-to-vigorous physical activity. Two studies of Kirk and coll. compared the effects of one-to-one physical activity counselling with the usual care in a 12 months research design. They found that the intervention group increased the level of physical activity, in respect of the control group participants [33]; furthermore, the authors reported a mean difference of 115 minutes/week of total physical activity in favor of the intervention group and a higher active stage of people who attended the intervention group in respect to the control group [34]. The Look Ahead Group in their 96 months trial, consisting of group and individual counselling on physical activity and reductions of fat intake, demonstrated an increase of the moderate intensity physical activity in the intervention group and a double total energy expenditure of the participants of the intervention group in comparison with the control participants. Plotnikoff et al. [37] described the effectiveness of a diabetes education program and an individualized physical activity counselling, they found an increase of the weekly moderate and vigorous activity as well as a higher rate of participants who achieved the physical activity recommendations of the intervention group in comparison with participants enrolled in diabetes education program only. Finally, Wadden and colleagues [39] found that 12 months of the intervention proposed in the Look Ahead Study [35] increase the physical activity level, and that, this increase is correlated with the weight loss of participants.

Despite the long list of studies reporting the effectiveness of counselling interventions on physical activity level, other researches [19] [23] [29] [32] [36] [38] reported the absence of positive effects or just slight but not significant results. Specifically, Batik, et al. [19] reported a general increase of physical activity levels among participants to the intervention, however, no statistically significant changes were found. The study of Debussche [23] showed a slight enhancement in sports activity in the intervention group, but no significant differences between the two groups were detected, as well as other differences concerning physical activity scores. Fowles and coll. [29] did not find statistically changes in clinical outcomes, however they found that subjects who were not meeting the physical activity guidelines increase their physical activity levels during the project; on the contrary, the more active participants in baseline, showed a decrease of physical activity levels during time. The work of Kirk, et al. [32] reported that the proposed interventions (physical activity consultation deli- 
vered by a person or in written form) were better, in terms of increasing the physical activity levels, than the usual care practice. Plotnikoff and coll. [36] did not found changes in physical activity levels in the intervention group, however, step counts increased in the female population who attended telephone counselling. Finally, Plotnikoff, et al. [38] reported no changes in physical activity levels after a 12 weeks intervention in a small sample of individuals with type 2 diabetes.

HbA1c change. Among the 17 studies reporting data on HbA1c, 9 (52.9\%) revealed a significant decrease in the counselling intervention group, while 7 (41.2\%) did not found any statistically significant change and 1 (5.9\%) reported a significant change in the comparison group rather than the counselling group.

Specifically, Belalcazar and colleagues [20] found a decrease of $0.7 \%$ in HbA1C in the intervention group, which was enrolled in-group and individual counselling program for 12 months. The control group presented anHbA1c decrease of $0.2 \%$, and the difference between groups was statistically significant. DeGreef et al. [22] demonstrated that their intervention based on a group counselling led by a behavioral expert or a general practitioner was effective in terms of HbA1c reduction in comparison with the usual care group. Otherwise, Debussche and coll. [23] found that both a counselling intervention of 4 sessions per year and a single visit in a year produced a within group significant decrease of HbA1c, however, no between groups interaction was detected. DiLoreto et al. [14] found an HbA1c significant change of -0.7 in the intervention group, while the control group did not show any changes. Furthermore, they highlighted that the reduction of HbA1c was related to an increase of energy expenditure. In the work of Fowles and colleagues [28] just one of the three intervention groups decreased significantly HbA1c levels; specifically the group trained by the kinesiologist had a significant effect, while the other groups did not show any substantial changes. Kang et al. [31] found that the intervention group involved for 24 months in the intervention showed significant lower HbA1c level in comparison to the control group. Moreover, they found statistically significant differences within the one-year and two-year intervention groups. Kirk and colleagues [34], in their first study, found statistically significant difference between groups, with a $0.26 \% \mathrm{HbA1c}$ decrease in the intervention group and a $0.15 \%$ increase in the control group. However, in their subsequent study [32], they demonstrated a main effect on HbA1c after 6 months of intervention, nevertheless no interactions between groups and time was found in their study. Finally, Yoo et al. [40] presented a difference between intervention and control group in post-intervention HbA1c levels. Specifically, in the intervention group $\mathrm{HbA} 1 \mathrm{c}$ decreased of $0.91 \%$ and $0.65 \%$ at 6 and 9 months from baseline, while the control group revealed an increase of $0.6 \%$ at 6 months and $0.25 \%$ at 9 months from baseline.

Also in the case of HbA1c, some studies [19] [21] [26] [27] [29] [36] did not found any changes between or within groups. More specifically, Batik, et al. [19] reported no significant change in mean HbA1c for the intervention group compared with controls. The study of Christian [21] showed that the $59 \%$ of the intervention patients experienced reductions in HbA1c level. However, this reduction was not statistically significant. Eakin and coll. [26] [27] did not find statistically changes in HbA1c levels during time, as well as Fowles and coll. [29] and Plotnikoff and coll. [36].

In one case [37], the authors found a decrease of $0.5 \%$ in HbA1c in the diabetes education group and no differences in the diabetes education group plus individual counselling. However, no differences between groups were found.

\section{Discussion}

Physical activity counselling may be a powerful solution in the management and treatment of patients with type 2 diabetes. This is because counselling strategies may act on the behavioral and psychological domains of the people, motivating them to be more and more engaged in physically demanding activities, overcoming internal barriers of sedentary lifestyle and providing better health status and trends [13]-[15].

The results presented in this systematic review seem to support the usefulness of physical counselling interventions on physical activity and HbA1c reduction in subjects with type 2 diabetes mellitus. However, not the totality of the studies reports positive effects on these two main outcomes. Because of this contradictory findings and great heterogeneity of study designs and interventions, a detailed discussion of findings is needed.

Regarding the associations between the interventions and physical activity level, scholars reporting positive and significant results proposed generally multi-component interventions in which the counselling strategies were coupled with other activities, such as therapeutic prescriptions, educational strategies etc. Specifically, 11 of 13 (84.6\%) articles reported this condition, while only the studies of Kirk and colleagues [33] [34] used just a 
physical activity-based counselling intervention. Regarding the interventions, it is also to notice that the majority of the studies, 8 of 13 (61.5\%), used a dietary co-intervention, focusing both on physical activity and nutritional education. In the majority of cases the control condition ( 7 of 13, 53.8\%) was represented by usual care activities; while in 5 of 13 studies (38.5\%) was partly involved in the activities proposed to the intervention group. In example, DiLoreto and colleagues [14] applied also for the control group a counselling activity on diet, physical activity and therapeutic prescription, but not the structured counselling proposed just to the intervention group. Similar strategies have been applied in other studies [35] [37] [39].

The duration of the intervention varied from the 3 months proposed by De Greef [22] to the 96 months of the Look Ahead Group study [35].

Finally, the quality level of these papers (intended as total number of "Yes" collected in the quality assessment; see Table 2) varied from 2 to 5 (mean $2.9 \pm 1.0$ ).

From the analysis, the great variety of type and duration of the interventions as well as quality of the studies was highlighted. Taking into account only papers reporting the effectiveness of the proposed interventions in terms of physical activity, it is not possible to state that in general counselling activities have a direct and positive influence. This is mainly because it is not possible to estimate the independent role of physical activity counselling on the outcome. Furthermore, lack of homogeneity in the types of control groups, duration of the programs and quality of the studies are other important sources of uncertainty in the definition of the real effectiveness of physical activity counselling interventions.

In regards to the associations between the interventions and HbA1c decrease, studies finding positive results proposed in the majority of times (6 of 9, 66.7\%) a multi-component intervention. In this case, also Debussche and coll. [23], in addition to Kirk and colleagues [33] [34], decided to test single role of physical activity counselling on the outcome. The dietary co-intervention has been used 5 times (55.6\%). The control group was based on usual care activities on 5 of 9 studies (55.6\%), while the remaining times was partly involved in the activities proposed to the intervention group. In this case, the duration of the intervention varied from 3 [22] to 24 [14] [32] months.

The quality of the studies analyzing HbA1c trends has been evaluated between 2 and 5, with a mean value of $2.9( \pm 1.3)$.

Among the studies reporting positive effects on HbA1c, limitations can be found in regards of the interventions. These limitations do not allow a clear and precise analysis of the real effectiveness of counselling strategies on HbA1c decrease.

Starting from the considerations about the lack of homogeneity in the interventions strategies, it is not possible to estimate, at now, the real effectiveness of a physical activity counselling intervention on physical activity level and HbA1c decrease.

In order to understand this possible relation, the role of the physical activity counselling on health outcomes need to be tested in an independent manner, avoiding the major sources of bias emerged from this review such as the co-interventions or the great diversity in the duration of the program and frequency of the sessions.

To define, develop, apply, validate and test an accurate and specific intervention might allow understanding the independent and causal role of physical counselling on health outcomes in type 2 diabetes mellitus patients.

The main strength of this study is represented by the focus on interventions studies reporting the use of a physical activity counselling strategy. The selection procedure allowed to include in the review just the articles that clearly reported the application of this kind of interventions, avoiding to enlarge the area of interest to the great variety of motivational and psychological interventions mainly focused on general lifestyle changes, diet, self-efficacy etc. The results of the study identify the current knowledge on the selected topic, directing future research to overcome the limitations described.

The first limitation of the current article may be detected in the inclusion of all the interventions studies, without a selection of just the randomized controlled trials. This choice was necessary, in this phase, to have a satisfactory number of papers to be analyzed. A second limitation can be found in the absence of statistical methods to compare the data from different papers, and the relative impossibility to conduct a meta-analysis, that may guarantee more precise and indicative results. However, in this stage, it was preferred, since the great variety of the identified studies, to analyze the results of the scientific articles with a descriptive approach. Finally, the third limitation can be seen as the analysis of just two outcomes (physical activity levels, HbA1c). The plethora of outcomes used in the selected studies would allow a more expanded selection, however, due to the lack of homogeneity of the studies it was decided to focus just on two of the main common and important health 
outcomes in type 2 diabetes mellitus. Further literature reviews may be oriented to analyze the effects of the interventions on a wider set of outcomes.

\section{Conclusion}

This systematic review aimed to analyze the role of physical activity counselling on physical activity level and HbA1c trend in patients with type 2 diabetes. The 23 interventional papers included in the review seem to report a general and slight positive effect, since, on average $60.7 \%$ of the papers report positive effects of the interventions on the outcomes. However, the lack of homogeneity and the great variability of the interventions do not allow understanding the independent role of the physical counselling on the selected outcomes. The contrasting results and the lack of homogeneity in the intervention protocols limit the possibility to affirm the usefulness of such an approach in patient with type 2 diabetes. In conclusion, further studies are needed to test the independent role of physical activity counselling on health outcomes in type 2 diabetes mellitus patients.

\section{Acknowledgements}

This study was made under the project "Counseling Motorio ed Attività Fisica Adattata quali azioni educativoformative perridefinire il percorso terapeutico e migliorare la qualità di vita del paziente con diabete mellito tipo 2" led by Prof. Giorgio Liguori, Università degli Studi di Napoli "Parthenope", and funded by the program "Centro Nazionale per la Prevenzione d il Controllo delle Malattie, 2012".

The authors would like to thank Prof. Giorgio Liguori for the scientific support and collaboration during the project.

\section{References}

[1] Thomas, D.E., Elliott, E.J. and Naughton, G.A. (2006) Exercise for Type 2 Diabetes Mellitus. Cochrane Database of Systematic Review, 19, CD002968. http://dx.doi.org/10.1002/14651858.CD002968.pub2

[2] Hayashino, Y., Jackson, J.L., Hirata, T., Fukumori, N., Nakamura, F., Fukuhara, S., Tsujii, S. and Ishii, H. (2014) Effects of Exercise on C-Reactive Protein, Inflammatory Cytokine and Adipokine in Patients with Type 2 Diabetes: A Meta-Analysis of Randomized Controlled Trials. Metabolism, 63, 431-440. http://dx.doi.org/10.1016/j.metabol.2013.08.018

[3] Thent, Z.C., Das, S. and Henry, L.J. (2013) Role of Exercise in the Management of Diabetes Mellitus: The Global Scenario. PLoS ONE, 8, e80436. http://dx.doi.org/10.1371/journal.pone.0080436

[4] Mikus, C.R., Oberlin, D.J., Libla, J., Boyle, L.J. and Thyfault, J.P. (2012) Glycaemic Control Is Improved by 7 Days of Aerobic Exercise Training in Patients with Type 2 Diabetes. Diabetologia, 55, 1417-1423. http://dx.doi.org/10.1007/s00125-012-2490-8

[5] Yavari, A., Hajiyev, A.M. and Naghizadeh, F. (2010) The Effect of Aerobic Exercise on Glycosylated Hemoglobin Values in Type 2 Diabetes Patients. The Journal of Sports Medicine and Physical Fitness, 50, 501-505.

[6] Reid, R.D., Tulloch, H.E., Sigal, R.J., Kenny, G.P., Fortier, M., McDonnell, L., Wells, G.A., Boulé, N.G., Phillips, P. and Coyle, D. (2010) Effects of Aerobic Exercise, Resistance Exercise or Both, on Patient-Reported Health Status and Well-Being in Type 2 Diabetes Mellitus: A Randomised Trial. Diabetologia, 53, 632-640. http://dx.doi.org/10.1007/s00125-009-1631-1

[7] Egger, A., Niederseer, D., Diem, G., Finkenzeller, T., Ledl-Kurkowski, E., Forstner, R., Pirich, C., Patsch, W., Weitgasser, R. and Niebauer, J. (2013) Different Types of Resistance Training in Type 2 Diabetes Mellitus: Effects on Glycaemic Control, Muscle Mass and Strength. European Journal of Preventive Cardiology, 20, 1051-1060. http://dx.doi.org/10.1177/2047487312450132

[8] Marcus, R.L., Smith, S., Morrell, G., Addison, O., Dibble, L.E., Wahoff-Stice, D. and Lastayo, P.C. (2008) Comparison of Combined Aerobic and High-Force Eccentric Resistance Exercise with Aerobic Exercise Only for People with Type 2 Diabetes Mellitus. Physical Therapy, 88, 1345-1354. http://dx.doi.org/10.2522/ptj.20080124

[9] Church, T.S., Blair, S.N., Cocreham, S., Johannsen, N., Johnson, W., Kramer, K., Mikus, C.R., Myers, V., Nauta, M., Rodarte, R.Q., Sparks, L., Thompson, A. and Earnest, C.P. (2010) Effects of Aerobic and Resistance Training on Hemoglobin A1c Levels in Patients with Type 2 Diabetes: A Randomized Controlled Trial. The Journal of American Medical Association, 304, 2253-2262. http://dx.doi.org/10.1001/jama.2010.1710

[10] American Diabetes Association (2011) Standards of Medical Care in Diabetes_-2011. Diabetes Care, 34, S11-S61. http://dx.doi.org/10.2337/dc11-S011 
[11] Colberg, S.R., Sigal, R.J., Fernhall, B., Regensteiner, J.G., Blissmer, B.J., Rubin, R.R., Chasan-Taber, L., Albright, A.L. and Braun, B. (2010) Exercise and Type 2 Diabetes: The American College of Sports Medicine and the American Diabetes Association: Joint Position Statement. Diabetes Care, 33, e147-167. http://dx.doi.org/10.2337/dc10-9990

[12] Thomas, N., Alder, E. and Leese, G.P. (2004) Barriers to Physical Activity in Patients with Diabetes. Postgraduate Medical Journal, 80, 287-291. http://dx.doi.org/10.1136/pgmj.2003.010553

[13] Korkiakangas, E.E., Alahuhta, M.A. and Laitinen, J.H. (2009) Barriers to Regular Exercise among Adults at High Risk or Diagnosed with Type 2 Diabetes: A Systematic Review. Health Promotion International, 24, 416-427. http://dx.doi.org/10.1093/heapro/dap031

[14] Di Loreto, C., Fanelli, C., Lucidi, P., Murdolo, G., De Cicco, A., Parlanti, N., Santeusanio, F., Brunetti, P. and De Feo, P. (2003) Validation of a Counseling Strategy to Promote The Adoption and the Maintenance of Physical Activity by Type 2 Diabetic Subjects. Diabetes Care, 26, 404-408. http://dx.doi.org/10.2337/diacare.26.2.404

[15] Doherty, Y. and Roberts, S. (2002) Motivational Interviewing in Diabetes Practice. Diabetic Medicine, 19, 1-6. http://dx.doi.org/10.1046/j.1464-5491.19.s3.2.x

[16] Moher, D., Liberati, A., Tetzlaff, J. and Altman, D.G., The PRISMA Group (2009) Preferred Reporting Items for Systematic Reviews and Meta-Analyses: The PRISMA Statement. PLoS Medicine, 6, e1000097. http://dx.doi.org/10.1371/journal.pmed.1000097

[17] Liberati, A., Altman, D.G., Tetzlaff, J., Mulrow, C., Gøtzsche, P.C., Ioannidis, J.P.A., Clarke, M., Devereaux, P.J., Kleijnen, J. and Moher, D. (2009) The PRISMA Statement for Reporting Systematic Reviews and Meta-Analyses of Studies That Evaluate Health Care Interventions: Explanation and Elaboration. PLoS Medicine, 6, e1000100. http://dx.doi.org/10.1371/journal.pmed.1000100

[18] Umpierre, D., Ribeiro, P.A.B., Kramer, C.K., Leitão, C.B., Zucatti, A.T.N., Azevedo, M.J., Gross, J.L., Ribeiro, J.P. and Schaan, B.D. (2011) Physical Activity Advice Only or Structured Exercise Training and Association with $\mathrm{HbA}_{1 \mathrm{c}}$ Levels in Type 2 Diabetes: A Systematic Review and Meta-Analysis. The Journal of American Medical Association, 305, 1790-1799. http://dx.doi.org/10.1001/jama.2011.576

[19] Batik, O., Phelan, E.A., Walwick, J.A., Wang, G. and LoGerfo, J.P. (2008) Translating a Community-Based Motivational Support Program to Increase Physical Activity among Older Adults with Diabetes at Community Clinics: A Pilot Study of Physical Activity for a Lifetime of Success (PALS). Preventing Chronic Disease, 5, A18.

[20] Belalcazar, L.M., Reboussin, D.M., Haffner, S.M., Hoogeveen, R.C., Kriska, A.M., Schwenke, D.C., Tracy. R.P., Pi-Sunyer, F.X., Ballantyne, C.M. and for the Look AHEAD Research Group (2010) A 1-Year Lifestyle Intervention for Weight Loss in Individuals with Type 2 Diabetes Reduces High C-Reactive Protein Levels and Identifies Metabolic Predictors of Change from the Look AHEAD (Action for Health in Diabetes) Study. Diabetes Care, 33, 2297-2303. http://dx.doi.org/10.2337/dc10-0728

[21] Christian, J.G., Bessesen, D.H., Byers, T.E., Christian, K.K., Goldstein, M.G. and Bock, B.C. (2008) Clinic-Based Support to Help Overweight Patients with Type 2 Diabetes Increase Physical Activity and Lose Weight. Archives of Internal Medicine, 168, 141-146. http://dx.doi.org/10.1001/archinternmed.2007.13

[22] De Greef, K., Deforche, B., Tudor-Locke, C. and De Bourdeaudhuij, I. (2011) Increasing Physical Activity in Belgian Type 2 Diabetes Patients: A Three-Arm Randomized Controlled Trial. International Journal of Behavioral Medicine, 18, 188-198. http://dx.doi.org/10.1007/s12529-010-9124-7

[23] Debussche, X., Rollot, O., Le Pommelet, C., Fianu, A., Le Moullec, N., Régnier, C., Boyer, M.C., Cogne, M., Bakiri, F., Schwager, J.C. and Favier, F. (2012) Quarterly Individual Outpatients Lifestyle Counseling after Initial Inpatients Education on Type 2 Diabetes: The REDIA Prev-2 Randomized Controlled Trial in Reunion Island. Diabetes \& Metabolism, 38, 46-53. http://dx.doi.org/10.1016/j.diabet.2011.07.002

[24] Eakin, E., Reeves, M., Winkler, E., Lawler, S. and Owen, N. (2010) Maintenance of Physical Activity and Dietary Change Following a Telephone-Delivered Intervention. Health Psychology, 29, 566-573. http://dx.doi.org/10.1037/a0021359

[25] Eakin, E., Reeves, M., Lawler, S., Graves, N., Oldenburg, B., Del Mar, C., Wilke, K., Winkler, E. and Barnett, A. (2009) Telephone Counseling for Physical Activity and Diet in primary Care Patients. American Journal of Preventive Medicine, 36, 142-149. http://dx.doi.org/10.1016/j.amepre.2008.09.042

[26] Eakin, E., Winkler, E.A., Dunstan, D.W., Healy, G.N., Owen, N., Marshall, A.M., Graves, N. and Reeves, M. (2014) Living Well with Diabetes: 24-Month Outcomes from a Randomized Trial of Telephone-Delivered Weight Loss and Physical Activity Intervention to Improve Glycemic Control. Diabetes Care, 37, 2177-2185. http://dx.doi.org/10.2337/dc13-2427

[27] Eakin, E.G., Reeves, M.M., Winkler, E., Healy, G.N., Dunstan, D.W., Owen, N., Marshal, A.M. and Wilkie, K.C. (2013) Six-Month Outcomes from Living Well with Diabetes: A Randomized Trial of a Telephone-Delivered Weight Loss and Physical Activity Intervention to Improve Glycemic Control. Annals of Behavioral Medicine, 46, 193-203. http://dx.doi.org/10.1007/s12160-013-9498-2 
[28] Fowles, J.R., Shields, C., d’Entremont, L., McQuaid, S., Barron, B. and Dunbar, P. (2014) Implementation of Resources to Support Patient Physical Activity through Diabetes Centres in Nova Scotia: The Effectiveness of Enhanced Support for Exercise Participation. Canadian Journal of Diabetes, 38, 423-431. http://dx.doi.org/10.1016/j.jcjd.2014.02.008

[29] Fowles, J.R., Shields, C., Barron, B., McQuaid, S. and Dunbar, P. (2014) Implementation of Resources to Support Patient Physical Activity through Diabetes Centres in Atlantic Canada: The Effectiveness of Toolkit-Based Physical Activity Counselling. Canadian Journal of Diabetes, 38, 415-422. http://dx.doi.org/10.1016/j.jcjd.2014.02.020

[30] Gregg, E.W., Chen, H., Wagenknecht, L.E., Clark, J.M., Delahanty, L.M., Bantle, J., Pownall, H.J., Johnson, K.C., Safford, M.M., Kitabchi, A.E., Pi-Sunyer, F.X., Wing, R.R., Bertoni, A.G. and for the Look AHEAD Research Group (2012) Association of an Intensive Lifestyle Intervention with Remission of Type 2 Diabetes. The Journal of American Medical Association, 308, 2489-2496. http://dx.doi.org/10.1001/jama.2012.67929

[31] Kang, J.Y., Cho, S.W., Sung, S.H., Park, Y.K., Paek, Y.M. and Choi, T.I. (2010) Effect of a Continuous Diabetes Lifestyle Intervention Program on Male Workers in Korea. Diabetes Research and Clinical Practice, 90, $26-33$. http://dx.doi.org/10.1016/j.diabres.2010.06.006

[32] Kirk, A., Barnett, J., Leese, G. and Mutrie, N. (2009) A Randomized Trial Investigating the 12-Month Changes in Physical Activity and Health Outcomes Following a Physical Activity Consultation Delivered by a Person or in Written form in Type 2 Diabetes: Time2Act. Diabetic Medicine, 26, 293-301. http://dx.doi.org/10.1111/j.1464-5491.2009.02675.x

[33] Kirk, A., Mutrie, N., MacIntyre, P. and Fisher, M. (2004) Effects of a 12-Month Physical Activity Counselling Intervention on Glycaemic Control and on the Status of Cardiovascular Risk Factors in People with Type 2 Diabetes. Diabetologia, 47, 821-832. http://dx.doi.org/10.1007/s00125-004-1396-5

[34] Kirk, A.F., Mutrie, N., Macintyre, P.D. and Fisher, M.B. (2004) Promoting and Maintaining Physical Activity in People with Type 2 Diabetes. American Journal of Preventive Medicine, 27, 289-296. http://dx.doi.org/10.1016/j.amepre.2004.07.009

[35] The Look AHEAD Research Group (2014) Eight-Year Weight Losses with an Intensive Lifestyle Intervention: The Look AHEAD Study: 8-Year Weight Losses in Look AHEAD. Obesity, 22, 5-13. http://dx.doi.org/10.1002/oby.20662

[36] Plotnikoff, R.C., Karunamuni, N., Courneya, K.S., Sigal, R.J., Johnson, J.A. and Johnson, S.T. (2013) The Alberta Diabetes and Physical Activity Trial (ADAPT): A Randomized Trial Evaluating Theory-Based Interventions to Increase Physical Activity in Adults with Type 2 Diabetes. Annals of Behavioral Medicine, 45, 45-56. http://dx.doi.org/10.1007/s12160-012-9405-2

[37] Plotnikoff, R.C., Pickering, M.A., Glenn, N., Doze, S.L., Reinbold-Matthews, M.L., McLeod, L.J., Lau, D.C., Fick, G.H., Johnson, S.T. and Flaman, L. (2011) The Effects of a Supplemental, Theory-Based Physical Activity Counseling Intervention for Adults with Type 2 Diabetes. Journal of Physical Activity \& Health, 8, 944-954.

[38] Plotnikoff, R.C., Johnson, S.T., Luchak, M., Pollock, C., Holt, N.L., Leahy, A., Liebreich, T., Sigal, R.J. and Boulé, N.G. (2010) Peer Telephone Counseling for Adults with Type 2 Diabetes Mellitus: A Case-Study Approach to Inform the Design, Development, and Evaluation of Programs Targeting Physical Activity. The Diabetes Educator, 36, 717-729. http://dx.doi.org/10.1177/0145721710376327

[39] Wadden, T.A., West, D.S., Neiberg, R.H., Wing, R.R., Ryan, D.H., Johnson, K.C., Foreyt, J.P., Hill, J.O., Trence, D.L. and Vitolins, M.Z., Look AHEAD Research Group (2009) One-Year Weight Losses in the Look AHEAD Study: Factors Associated With Success. Obesity, 17, 713-722. http://dx.doi.org/10.1038/oby.2008.637

[40] Yoo, J.S., Lee, S.J., Lee, H.C. and Kim, M.J. (2007) The Effect of a Comprehensive Lifestyle Modification Program on Glycemic Control and Body Composition in Patients with Type 2 Diabetes. Asian Nursing Research, 1, 106-115. http://dx.doi.org/10.1016/S1976-1317(08)60013-4

\section{Appendix}

((((“motor activity” [MeSH Terms] OR (“motor” [All Fields] AND “activity” [All Fields]) OR "motor activity” [All Fields] OR ("physical” [All Fields] AND "activity” [All Fields]) OR "physical activity” [All Fields]) AND (“counselling” [All Fields] OR “counseling” [MeSH Terms] OR "counseling” [All Fields])) OR ((“physical examination” [MeSH Terms] OR ("physical” [All Fields] AND “examination” [All Fields]) OR "physical examination” [All Fields] OR "physical” [All Fields]) AND (“counselling” [All Fields] OR "counseling” [MeSH Terms] OR "counseling” [All Fields]))) OR (“counselling” [All Fields] OR "counseling” [MeSH Terms] OR “counseling” [All Fields])) AND (“diabetes mellitus, type 2” [MeSH Terms] OR “type 2 diabetes mellitus” [All Fields] OR "type 2 diabetes” [All Fields]) 